\section{MS34-O5 Applications of density functional theory to crystal structure solution for multicomponent crystals}

David S. Edgeley ${ }^{1}$, Kenneth Shankland ${ }^{1}$, Chris S. Frampton ${ }^{2}$

1. Univeristy of Reading

2. Brunel Universtiy, London

email: d.s.edgeley@pgr.reading.ac.uk

Analysis of crystal structures in the Cambridge Structural Database (CSD) shows that there is an upwards trend in the number of structures being solved using X-ray powder diffraction, in spite of its limitations relative to single-crystal diffraction data. This trend can be attributed to the increasing quality of the diffraction data being collected, the ongoing development of techniques for solving structures, inherent interest in polycrystalline systems and ever-increasing computing power. Yet X-ray powder diffraction remains (in general) too information-poor in order to reliably determine hydrogen atom positions, and this affects conclusions that can be drawn from the crystal structure e.g. is a multicomponent system co-crystal or a salt ${ }^{1}$ ?

The FDA defines pharmaceutical cocrystals and salts differently and regulatory schemes governing their production and identification differ significantly. Currently, cocrystals are defined as "crystalline materials composed of two or more molecules within the same crystal lattice" and generally classified by having a $\Delta \mathrm{p} K_{\mathrm{a}}$ $<1$, but they do not have a regulatory scheme. Pharmaceutical salts are highly regulated and defined as "Any of numerous compounds that result from replacement of part of all of the acid hydrogen of an acid by a metal or a radical acting like a metal: an ionic or electrovalent crystalline compound." Unfortunately the chemical difference between a salt and a cocrystal is far from distinct; a continuum of hydrogen atom transfer situations exists.

Pyrimethanil, a broad spectrum fungicide, has a tendency to form multicomponent crystal structures with lattices constructed from intermolecular bonds H-bonds lying along the cocrystal-salt continuum. Here we demonstrate that dispersion-corrected density functional theory (DFT-D) ${ }^{2}$ can distinguish between pyrimethanthil salts, cocrystals and intermediates by precisely determining hydrogen position. We then show how periodic DFT has been integrated into our structure determination workflows and demonstrate the advantage of this approach in accurately determining multicomponent crystal structures.

1. Chan, H.C.S., Kendrick, J., Neumann, M.A. \& Leusen, F.J.J. Crystengcomm 15, 3799-3807 (2013).

2. Giannozzi, P. et al. Journal of Physics-Condensed Matter 21 (2009).

Keywords: Salt, Cocrystal, DFT, H-bond
MS35. Dynamics in

nanoporous molecular crystals
Chairs: Angiolina Comotti, Len Barbour

\section{MS35-O1 Breathing porous molecular crystals}

Kari Rissanen ${ }^{1}$

1. Department of Chemistry, Nanoscience Center, University of Juväskylä, Jyväskylä, 40014, Finland)

email: kari.t.rissanen@jyu.fi

An exciting research challenge in crystal engineering and supramolecular chemistry is to design, synthesize, and characterize porous architectures ${ }^{1}$, topical example being the metallo-organic frameworks, MOF's, with applications in catalysis, separation, trapping of gases, etc. $^{2}$ The design of porous lattices normally relies on rigid 3-D lattices, which in some cases can undergo single-crystal-to-single-crystal transformations ${ }^{3}$ or exhibit porous behavior in a seemingly non-porous crystals. ${ }^{4}$

Our research interests over the last 25 years have been focused on the studies of weak non-covalent intermolecular, viz. supramolecular interactions as the driving force in self-assembly and molecular recognition, especially in the solid state by single crystal X-ray diffraction. Particularly the hierarchy and interplay of interactions, viz. concerted interactions, are becoming more and more important in the understanding of molecular self-assembly and crystal growth. Recently our work on halogen bonding ${ }^{5}$ revealed a halogen and hydrogen bonded (XB and $\mathrm{HB}$ ) system based on an XB complex hexamethylenetetramine (HMTA) and $\mathrm{N}$-iodosuccinimide (NIS) ${ }^{6}$ to result in a porous, yet breathing crystal lattice. The lecture will highlight the results and behavior of this intriguing system exhibiting concerted action of XB and HB.

\section{References}

1. L. J. Barbour, Chem. Commun. 2006, 1163.

2. Thematic issue on MOF's, Chem Soc. Rev. 2014, 43.

3. S. Sen, S. Neogi, K. Rissanen, P. K. Bharadwaj, Chem. Commun. 2015, 51, 3173.

4. S. A. Herbert, A. Janiak, P. K. Thallapally, J. L. Atwood, L. J. Barbour, Chem. Commun. 2014, 50, 15509.

5. G. R. Desiraju, P. S. Ho, L. Kloo, A. C. Legon, R. Marquardt, P. Metrangolo, P. A. Politzer, G. Resnati and K Rissanen, Pure Appl. Chem. 2013, 85, 1711.

6. K. Raatikainen and Kari Rissanen, CrystEngComm 2011, 13, 6972.

7. K. Raatikainen, K. Rissanen, Chem. Sci. 2012, 2, 235.

Keywords: crystal structure, porosity, halogen bonding, hydrogen bonding 